\title{
Simulation of Corrugated Coaxial Cables using the Meshless Radial Point Interpolation Time-Domain Method
}

\author{
Christoph Böcklin \#, Thomas Kaufmann \#, Johannes Hoffmann \#, Christophe Fumeaux *, Rüdiger Vahldieck \# \\ \# Laboratory for Electromagnetic Fields and Microwave Electronics (IFH), \\ Member of SEREC (Swiss Electromagnetics Research \& Engineering Centre), \\ ETH Zurich, 8092 Zurich, Switzerland \\ boecklicdee.ethz.ch \\ * School of Electrical \& Electronic Engineering, The University of Adelaide \\ Adelaide, South Australia 5005
}

\begin{abstract}
Meshless numerical simulation methods are gaining attention in different research fields. The ability for conformal and multi-scale modelling as well as the potential for a dynamic node adaptation make them interesting for many applications. The meshless Radial Point Interpolation Method (RPIM) in Time-Domain is proposed here as a versatile simulation technique for electromagnetic problems. In this framework, a conformal boundary treatment is described and validated in a $2 \mathrm{D}$ cylindrically symmetric arrangement. These conformal boundaries can be applied for example to simulate the influence of production tolerances or manufacturing properties in corrugated coaxial copper cables. Reflections arising from ripples of a corrugated copper cable are analyzed in particular here and the simulation results exhibit a perfectly linear dependence in a log-log plot between the reflected energy and the extent of the corrugation. The obtained results are validated successfully through comparison with a Finite-Element solution of the problem. Finally, the phase and amplitude variations of a sinusoidal signal propagating in the corrugated cable are characterized.
\end{abstract}

\section{INTRODUCTION}

Meshless methods have become increasingly popular in many engineering specialties over the past years [1]. They might grow to become a viable alternative to well-established simulation methods like the Finite-Element Method (FEM) or the Finite-Difference Time-Domain (FDTD) method. Meshless methods appear attractive as they offer a flexible treatment of geometries, but do not require a fixed mesh topology and can be applied in the framework of a simple explicit time-domain iteration. However, at present, the class of meshless methods poses many challenges as novel technique for the simulation of electromagnetic problems [2], [3]. The Radial Point Interpolation Method (RPIM) [4], [5] is a promising candidate among the different types of meshless methods for simulating such problems. RPIM can be applied to a staggered set of arbitrarily placed E- and H-nodes without requiring explicit connectivity among them. Radial basis functions (RBF) are used to interpolate the field values in between these nodes.

The possibility of placing the nodes at arbitrary locations allows for multi-scale simulations, conformal boundary treat- ment and in principle dynamic adaptation of the node arrangement. This flexibility as well as the possible application of the method for various partial differential equations make the RPIM a promising alternative to classical numerical methods for transient multi-physics applications, e.g. in nanotechnology [6], [7], [8].

This paper describes a 2D implementation of the RPIM method for cylindrically symmetric models and demonstrates its application to a practical transmission line problem. Corrugated copper cables are flexible air dielectric coaxial cables. They are widely used for high power applications, for example as connection cables for mobile base stations or terrestrial antennas. The corrugations facilitates physical handling, e.g. bending. However, the periodic variations of the profile also cause reflections of waves propagating in the cable. The computation of reflection in corrugated cables is shown here as illustration of one application in the field of electromagnetic compatibility (EMC) of the attractive properties of the meshless RPIM.

\section{THEORY}

\section{A. Meshless Methods}

Meshless methods differ from FEM or FDTD in that they don't need an explicit mesh topology or a regular grid to discretize the problem domain. They rather use a set of arbitrarily placed nodes and do not require an explicit connectivity among them. Around each node a support domain with radius $d_{n}$ is defined (see Fig. 1), containing a certain number of neighboring nodes. The vector field value of a node $n$ is approximated by an interpolation function constructed on the basis of the values from the nodes in this support domain. The size of the support domain can be in principle chosen freely. However, an optimal size will always be a trade-off between simulation speed, stability and numerical accuracy of the interpolation. The numerical accuracy can be increased through a refinement of the discretization, i.e. through a denser node distribution in the model. 


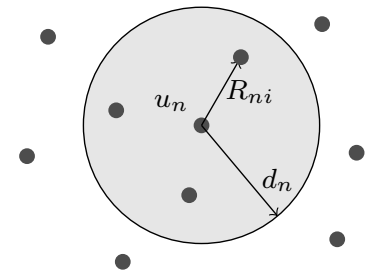

Figure 1. Illustration of the support domain with radius $d_{n}$ around the node $n$, with approximated field value $u_{n} . R_{n i}$ denotes the Euclidean distance between node $n$ and node $i$.

\section{B. The Meshless Radial Point Interpolation Method (RPIM)}

A generic function in the function space can be expressed as a linear combination of basis functions. The RPIM ([4], [5]) uses an interpolation based on radial basis functions $r_{i}$ and monomial basis functions $p_{j}$ [9] to approximate the function value $u_{n}$ of node $n$ (see Fig. 1) at the location $\mathbf{x}_{n}=\left(x_{n}, y_{n}\right)$ :

$$
\begin{aligned}
u\left(\mathbf{x}_{n}\right)=u_{n} \approx\left\langle u_{n}\right\rangle & =\sum_{i=1}^{N} r_{i}\left(\mathbf{x}_{n}\right) a_{i}+\sum_{j=1}^{M} p_{j}\left(\mathbf{x}_{n}\right) b_{j} \\
& =\mathbf{r}^{T}\left(\mathbf{x}_{n}\right) \mathbf{a}+\mathbf{p}^{T}\left(\mathbf{x}_{n}\right) \mathbf{b} .
\end{aligned}
$$

where $a_{i}$ and $b_{j}$ are the coefficients for the linear combination of the basis functions, $N$ denotes the number of nodes in the support domain of node $n$ and $M$ is the number of the monomial terms $p$. The vectors in (1) are defined as

$$
\begin{aligned}
\mathbf{a}^{T} & =\left[a_{1}, a_{2}, \ldots, a_{N}\right] \\
\mathbf{b}^{T} & =\left[b_{1}, b_{2}, \ldots, b_{M}\right] \\
\mathbf{r}^{T}\left(\mathbf{x}_{n}\right) & =\left[r_{1}\left(\mathbf{x}_{n}\right), r_{2}\left(\mathbf{x}_{n}\right), \ldots, r_{N}\left(\mathbf{x}_{n}\right)\right] \\
\mathbf{p}^{T}\left(\mathbf{x}_{n}\right) & =\left[p_{1}\left(\mathbf{x}_{n}\right), p_{2}\left(\mathbf{x}_{n}\right), \ldots, p_{M}\left(\mathbf{x}_{n}\right)\right] .
\end{aligned}
$$

A popular radial basis for the RPIM is the exponential Gaussian

$$
r_{i}\left(\mathbf{x}_{n}\right)=\exp \left(-c\left(R_{n i} / d_{n}\right)^{2}\right)
$$

with $R_{n i}=\left\|\mathbf{x}_{n}-\mathbf{x}_{i}\right\|$ being the Euclidean distance between node $n$ and $i$. The linear monomial basis is defined as

$$
\mathbf{p}^{T}\left(\mathbf{x}_{n}\right)=\left[\begin{array}{lll}
1 & x & y
\end{array}\right] \text {. }
$$

In order to obtain the coefficients $\mathbf{a}$ and $\mathbf{b}$ from (1), the following system of linear equations has to be solved for each node $n$ in the computational domain

$$
\begin{aligned}
\underbrace{\left[\begin{array}{cc}
\mathbf{R}_{0} & \mathbf{P}_{0} \\
\mathbf{P}_{0}^{T} & \mathbf{0}
\end{array}\right]}_{\mathbf{G}}\left\{\begin{array}{l}
\mathbf{a} \\
\mathbf{b}
\end{array}\right\}=\left\{\begin{array}{c}
\mathbf{u}^{e} \\
\mathbf{0}
\end{array}\right\} \\
\rightarrow\left\{\begin{array}{l}
\mathbf{a} \\
\mathbf{b}
\end{array}\right\}=\mathbf{G}^{-1}\left\{\begin{array}{c}
\mathbf{u}^{e} \\
\mathbf{0}
\end{array}\right\}
\end{aligned}
$$

where $\mathbf{u}^{e}$ is the vector for function values at the $N$ neighboring nodal points. The matrix $\mathbf{R}_{0}$ that multiplies the unknown coefficient vector $\mathbf{a}$, is constructed as

$$
\mathbf{R}_{0}=\left[\begin{array}{cccc}
r_{1}\left(\mathbf{x}_{\mathbf{1}}\right) & r_{2}\left(\mathbf{x}_{\mathbf{1}}\right) & \ldots & r_{N}\left(\mathbf{x}_{\mathbf{1}}\right) \\
r_{1}\left(\mathbf{x}_{\mathbf{2}}\right) & r_{2}\left(\mathbf{x}_{\mathbf{2}}\right) & \ldots & r_{N}\left(\mathbf{x}_{\mathbf{2}}\right) \\
\vdots & \vdots & \vdots & \vdots \\
r_{1}\left(\mathbf{x}_{\mathbf{N}}\right) & r_{2}\left(\mathbf{x}_{\mathbf{N}}\right) & \ldots & r_{N}\left(\mathbf{x}_{\mathbf{N}}\right)
\end{array}\right]_{N \times N}
$$

and the matrix $\mathbf{P}_{0}$, that is applied on the unknown coefficient vector $\mathbf{b}$ is

$$
\mathbf{P}_{0}=\left[\begin{array}{cccc}
p_{1}\left(\mathbf{x}_{\mathbf{1}}\right) & p_{2}\left(\mathbf{x}_{\mathbf{1}}\right) & \ldots & p_{M}\left(\mathbf{x}_{\mathbf{1}}\right) \\
p_{1}\left(\mathbf{x}_{\mathbf{2}}\right) & p_{2}\left(\mathbf{x}_{\mathbf{2}}\right) & \ldots & p_{M}\left(\mathbf{x}_{\mathbf{2}}\right) \\
\vdots & \vdots & \vdots & \vdots \\
p_{1}\left(\mathbf{x}_{\mathbf{N}}\right) & p_{2}\left(\mathbf{x}_{\mathbf{N}}\right) & \ldots & p_{M}\left(\mathbf{x}_{\mathbf{N}}\right)
\end{array}\right]_{N \times M} .
$$

The condition $\mathbf{P}_{0}^{T} \cdot \mathbf{a}=\mathbf{0}$ guarantees the uniqueness of the solution. The interpolation can finally be written as

$$
\left\langle u_{n}\right\rangle=\left[\begin{array}{ll}
\mathbf{r}^{T}\left(\mathbf{x}_{n}\right) & \mathbf{p}^{T}\left(\mathbf{x}_{n}\right)
\end{array}\right] \mathbf{G}^{-1}\left\{\begin{array}{c}
\mathbf{u}^{e} \\
\mathbf{0}
\end{array}\right\} \equiv \mathbf{\Psi}\left(\mathbf{x}_{n}\right) \mathbf{u}^{e} .
$$

This can be interpreted as a weighted sum of the field values at neighboring nodes, where the weights are determined by the shape function $\Psi\left(x_{n}\right)$. The approximation of the spatial derivative of the field values can be found by taking the derivative of the basis functions

$$
\left\langle\partial u_{n}\right\rangle=\left[\begin{array}{ll}
\partial \mathbf{r}^{T}\left(\mathbf{x}_{n}\right) & \partial \mathbf{p}^{T}\left(\mathbf{x}_{n}\right)
\end{array}\right] \mathbf{G}^{-1}\left\{\begin{array}{c}
\mathbf{u}^{e} \\
\mathbf{0}
\end{array}\right\}=\partial \mathbf{\Psi}\left(\mathbf{x}_{n}\right) \mathbf{u}^{e} .
$$

\section{Cylindrically Symmetric Maxwell's Equations for TM- Mode}

The RPIM makes use of a discretized version of the Maxwell's equations in differential form. Although corrugated copper cables usually have helical corrugations, they are assumed here to be rotationally symmetric along the cable axis. This assumption reduces the three dimensional problem to two dimensions, as only a radial plane has to be simulated. Rotationally symmetric Maxwell's equations can be obtained by formulation in cylindrical coordinates. The spatial discretization is obtained by using a staggered distribution of $\mathrm{E}$ - and $\mathrm{H}$-nodes and by replacing the field values and their derivatives by the approximations (8) and (9):

$$
\begin{aligned}
& \partial_{t} H_{\varphi, m}=\frac{1}{\mu}\left[\sum_{i=1}^{N_{m}} \partial_{r} \Psi_{i} \cdot E_{z, i}\right.\left.-\sum_{i=1}^{N_{m}} \partial_{z} \Psi_{i} \cdot E_{\rho, i}\right] \\
& \partial_{t} E_{\rho, n}=-\frac{1}{\varepsilon}\left[\sum_{i=1}^{N_{n}} \partial_{z} \Psi_{i} \cdot H_{\varphi, i}\right] \\
& \partial_{t} E_{z, n}=\frac{1}{\varepsilon}\left[\frac{1}{\rho(n)} \sum_{i=1}^{N_{n}} \Psi_{i} \cdot H_{\varphi, i}\right. \\
&\left.+\sum_{i=1}^{N_{n}} \partial_{r} \Psi_{i} \cdot H_{\varphi, i}\right] .
\end{aligned}
$$

Discretizing (10) in time, the update equations for the RPIM can be obtained by adopting a leap-frog time-stepping scheme for the temporal derivative. To assure a stable simulation, a stability criterion similar to the Courant-Friedrichs-Levy (CFL) limit in FDTD is used, which depends on the shortest distance between any two nodes:

$$
\Delta t \leq \min _{i} \frac{d_{\min , \mathrm{i}}}{c}
$$




\section{Conformal Boundaries}

A concept for the treatment of conformal boundaries in RPIM is introduced here. A material boundary of the model is not only characterized by its position but also by a normal vector $\vec{n}$, thus giving additional geometrical information required to model arbitrary shapes. The radially symmetric coaxial cable to be simulated has metal on both, the top and the bottom, which is assumed to be a perfect electric conductor (PEC), i.e. with the tangential component of the E-field at the boundary equal to zero (see Fig. 2).

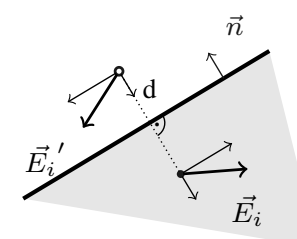

Figure 2. Field value of an E-node and its image at an arbitrarily oriented PEC boundary.

One way to enforce this condition is to place additional nodes outside of the model [10]. The positions of the additional PEC-nodes are obtained by mirroring the E-nodes that are closest to the boundary at the PEC. The field values of these added nodes are calculated in such a way, that averaging the electric field values of a node and its image results in a zero tangential field component (see Fig. 2). This condition can be expressed as

$$
\vec{E}_{i}^{\prime}=-\vec{E}_{i}+2\left(\vec{n} \cdot \vec{E}_{i}\right) \vec{n} .
$$

where the values $E_{i}^{\prime}$ in the mirror nodes are computed based on the values $E_{i}$ of the E-nodes at the current time step.

\section{Simulation SETUP}

\section{A. Models}

Figure 3 shows the models of the plain coaxial cable (Fig. 3 a)) and a cable with a corrugated part (b). The dimensions of the model are $D_{\text {outer }}=14.28 \mathrm{~cm}$ and $d_{\text {inner }}=7.14 \mathrm{~cm}$, respectively. These dimensions are close to those of commercially available high-power cables. The corrugations are assumed to be of a sinusoidal form with an amplitude varied between $0.0002 \%$ and $4 \%$ of the thickness of the dielectric. The total length of the corrugations is $l_{C}=14.28 \mathrm{~cm}$ and include 4.5 sinusoidal periods. These corrugations cause a part of the energy of an incoming wave to be reflected. The model
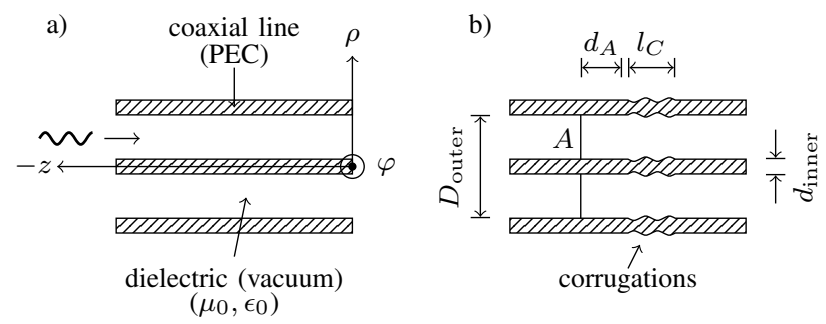

Figure 3. a) Plain and b) corrugated coaxial cable of a plain cable (a) provides a reference for the analysis of the simulation data. The bulk of the computational domain is filled by a staggered $\mathrm{E} / \mathrm{H}$ grid-like node distribution with a node spacing corresponding to $\lambda_{0} / 28$. The regular grid is disturbed near the corrugations. The simulation parameters are set to $c=0.6$ for the gaussian basis function (3). The radius of the support domain $d_{n}$ is set such that at least $N_{\text {min }}=6$ are contained in a support domain. The choice of these parameters is a trade-off between interpolation accuracy and computational cost, and a result of best practice.

\section{B. Excitation}

The fundamental mode propagating in a coaxial cable is a TEM mode with $\mathrm{E}$ - and $\mathrm{H}$-field perpendicular to the propagation direction. However, above the cut-off frequency higher-order modes can exist, which can be excited by the corrugations. For the mentioned cable dimensions, the cutoff frequency for rotational symmetric modes corresponds to $4.2 \mathrm{GHz}$ [11]. In order to prohibit the existence of higher-order modes, the frequency $\omega$ of the sinusoidal harmonic excitation $H_{\varphi}(\rho)=\frac{1}{\rho} a \cdot \sin (\omega t+c)$ is chosen to be $3 \mathrm{GHz}$, thus below the cut-off frequency.

\section{RESULTS}

\section{A. Computation of Reflection}

The reflections from the corrugations are computed by calculating

$$
\Gamma_{\mathcal{E}}=\frac{\mathcal{E}_{r}}{\mathcal{E}_{i}}
$$

where $\mathcal{E}_{r}$ and $\mathcal{E}_{i}$ are the reflected and incident energy flowing through a cable cross-section $A$ (see Fig. 3 ) over one period. The plane $A$ is placed $d_{A}=10 \mathrm{~cm}$ before the start of the corrugations. This is sufficiently far to ensure that evanescent higher-order modes have decayed enough and do not affect the computed energy significantly.

The energy flow integrated over one period is defined as

$$
\mathcal{E}=\int_{0}^{2 \pi} \int_{\frac{d_{\text {inner }}}{2}}^{\frac{D_{\text {outer }}}{2}} \underbrace{\frac{1}{T} \int_{0}^{T} E_{\rho}(\rho, t) \cdot H_{\varphi}(\rho, t) \mathrm{d} t}_{S_{a v}} \cdot \rho \mathrm{d} \rho \mathrm{d} \varphi
$$

$E_{\rho}(\rho, t)$ and $H_{\varphi}(\rho, t)$ are the field values at time $t$ and position $\rho$ in the plane $A$. The field distribution for the reflected wave is obtained by subtracting the reference solution obtained in the plain cable from the solution obtained in the corrugated cable. The energy of the incident wave correspond to the value obtained from the reference model. $S_{a v}$ is the time-averaged Poynting vector in $\mathrm{z}$ direction.

Figure 4 shows the linear dependence in a log-log plot between the reflection coefficient $\Gamma_{\mathcal{E}}$ and the amplitude of the sinusoidal corrugations of the cable. The calculations show that in cables with large corrugations a considerable amount of the incident energy is reflected. Corrugations of cables used in practical applications such as mobile base stations extend to as much as $4 \%$ of the thickness of the dielectric air gap. The resulting reflection coefficient is in this case $-26 \mathrm{~dB}$. The 
reflection coefficient converges to zero as the corrugations vanish. The calculations performed with an RPIM implementa-

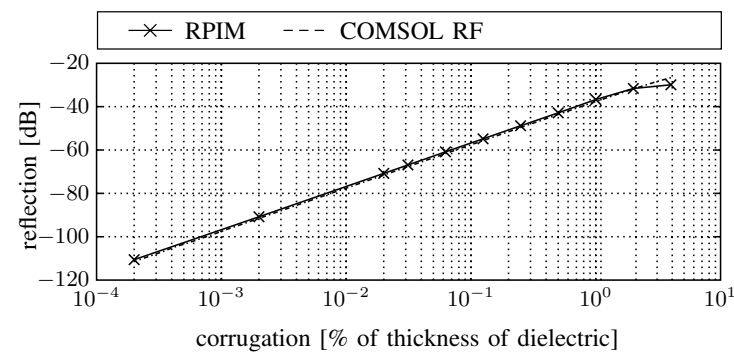

Figure 4. Comparison of the reflection coefficient $\Gamma_{\mathcal{E}}$ computed with RPIM and Comsol.

tion are compared with corresponding computations obtained with the commercial software COMSOL RF Module, based on the frequency-domain FEM. In the model defined in the commercial tool, the cable is terminated with ports, which in addition allow the direct extraction of the reflection coefficient. The distance from the ports to the corrugation section was varied until no influence of the higher-order mode was found, which confirmed the validity of the chosen distance $d_{A}=10$ $\mathrm{cm}$. Finally the reflected energy is computed as

$$
\Gamma_{\mathcal{E}}=\left|S_{11}\right|^{2} \text {. }
$$

The comparison shown in Fig. 4 demonstrate a a very good agreement between the two numerical methods.

\section{B. Amplitude and Phase Analyses}

Amplitude and phase analyses of the harmonic wave at 3 $\mathrm{GHz}$ were performed in the corrugated part of the cable. The amplitude of the corrugations for this investigation respresents $2 \%$ of the thickness of the dielectric. Figure 5 shows the amplitude and the phase of the standing wave pattern at the corrugation for the total field, i.e. including incident and reflected wave. The standing wave ratio in Section $\mathrm{A}_{1}$ (before the corrugations) characterizes the reflection coefficient. The maximal amplitude in section $\mathrm{A}_{3}$ (after the corrugations) is smaller than that of the reference, which is consistent with the fact that part of the energy is reflected.

Figure 5b) shows the phase difference between the plain cable reference model and the corrugated model. The phase variations in section $P_{1}$ is again consistent with the standing wave. The phase difference in section $\mathrm{P}_{3}$ is negative. This shows a retardation caused by a slightly increased characteristic impedance in the corrugated segment $\mathrm{P}_{2}$.

\section{CONClusion}

The meshless RPIM in Time-Domain is proposed as a versatile novel technique for numerical simulation of electromagnetic problems. A suitable boundary treatment for the RPIM has been implemented and applied on conformal boundaries. A model of a corrugated coaxial cable has been simulated with a cylindrically symmetric version of the RPIM and reflections arising from the diameter variations have been computed. The achieved results show very good agreement with reference calculations done with FEM simulations (COMSOL), validating

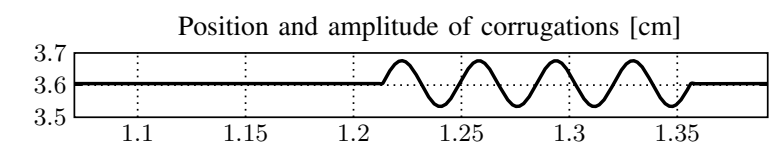

a) Maximal amplitude

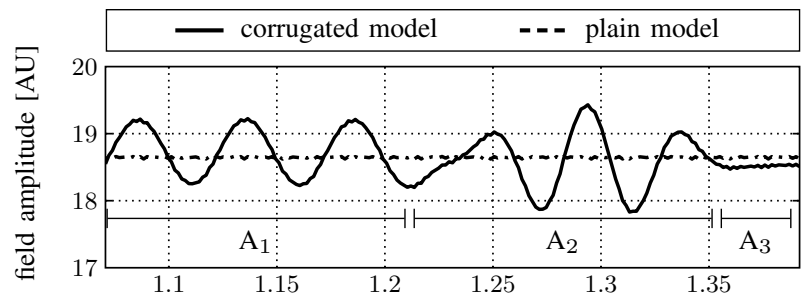

b) Phase difference

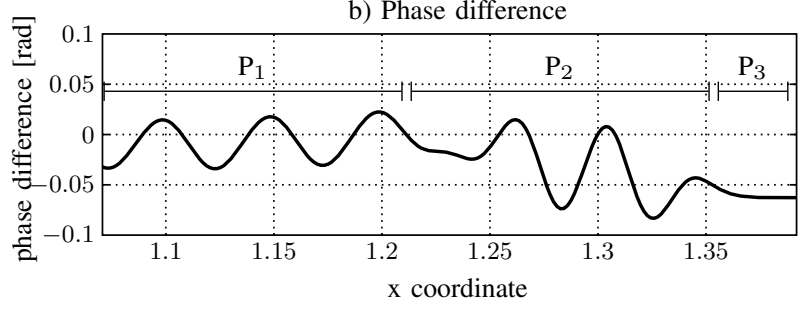

Figure 5. Amplitude variation and phase difference of the test model.

the conformal boundary treatment. The RPIM is in its infancy and more research is required, e.g. to extend the concepts for a full 3D application, or to assure long-term stability of the simulation (i.e. over several hundreds of periods).

\section{REFERENCES}

[1] G. Liu and Y. Gu, An introduction to meshfree methods and their programming. Springer, 2005.

[2] G. Ala, E. Francomano, A. Tortorici, E. Toscano, and F. Viola, "Smoothed Particle ElectroMagnetics: A mesh-free solver for transients," Journal of Computational and Applied Mathematics, vol. 191, no. 2, pp. 194-205, 2006.

[3] L. Balewski, A. Cwikla, P. Kowalczyk, P. Kozakowski, L. Kulas, A. Lamecki, M. Mrozowski, K. Nyka, P. Sypek, and M. Wiktor, "Advances in computational electromagnetics," 15th International Conference on Microwaves, Radar and Wireless Communications, MIKON2004., vol. 2, pp. 613-625, May 2004.

[4] J. Wang and G. Liu, "A point interpolation meshless method based on radial basis functions," Int. J. Numer. Meth. Eng, vol. 54, pp. 1623-1648, 2002.

[5] T. Kaufmann, C. Fumeaux, and R. Vahldieck, "Application of the Radial Point Interpolation Method as Meshless Time-Domain Technique in Electromagnetics," IEEE MTT-S International Microwave Symposium (IMS 2008), Atlanta, 2008.

[6] G. Liu and Y. Gu, "A local radial point interpolation method (LRPIM) for free vibration analyses of 2-D solids," Journal of Sound and Vibration, vol. 246, no. 1, pp. 29-46, 2001.

[7] G. Liu, K. Dai, K. Lim, and Y. Gu, "A radial point interpolation method for simulation of two-dimensional piezoelectric structures," Smart Materials and Structures, vol. 12, no. 2, pp. 171-180, 2003.

[8] G. Liu, G. Zhang, Y. Gu, and Y. Wang, "A meshfree radial point interpolation method (RPIM) for three-dimensional solids," Computational Mechanics, vol. 36, no. 6, pp. 421-430, 2005.

[9] M. Buhmann, Radial Basis Functions: Theory and Implementations. Cambridge University Press, 2003.

[10] K. Krohne, G.-H. Park, and L. E. Ping, "Waveguide Mode Computation through Smoothed Particle Electromagnetics," in 24th International Review of Progress in Applied Computational Electromagnetics (ACES 2008), Niagara Falls, Canada, April 2008, pp. 882-887.

[11] H. Meinke and F. Gundlach, Taschenbuch der Hochfrequenztechnik, 2nd ed. Springer, 1962, p. 309. 